\title{
Temporäre Beschaulichkeit um einen innovativen Kern
}

Bürgerstiftungen in Deutschland

\section{Stefan Nährlich}

\begin{abstract}
A citizens' foundation collects many, though small amounts of money to be used for an intentionally wide array of tasks defined by the foundation. It is concerned with the community of a city or a region. A common aspect of their self-understanding is their independence with regard to statutory, municipal and entrepreneurial structures. Whereas traditional foundations lack in many cases internal mechanisms of control, most of the citizens' foundations do have such mechanisms. The entire assets of such foundations in Germany currently amount to more than 60 million euro.
\end{abstract}

Une fondation citoyenne collecte beaucoup d'argent, même s'il s'agit de petites sommes, destiné à un éventail intentionnellement large de tâches, défini par les fondateurs. Elle cible généralement une ville ou une région. Une de leur caractéristique commune réside dans leur indépendance vis à vis des autorités publiques, municipales et des entreprises commerciales. Tandis que les fondations traditionnelles ne disposent pas la plupart $d u$ temps de mécanismes de contrôle internes, la plupart des fondations citoyennes en ont-elles instaurés. L'actif complet de telles fondations s'élève actuellement en Allemagne à environ 60 millions d'euros.

\section{Dr. Stefan Nährlich ist}

Geschäftsführer der Aktiven

Bürgerschaft, dem Kompetenzzentrum für Bürgerengagement der Volksbanken und Raiffeisenbanken.

E-Mail

stefan.naehrlich@aktive-buergerschaft.de
Eine Bürgerstiftung sammelt von Bürgern viele eher kleine Geldsummen zum Einsatz für einen möglichst breiten Stiftungszweck. Sie engagiert sich für das Gemeinwesen einer bestimmten Stadt oder Region. Zum Selbstverständnis der meisten Bürgerstiftungen gehört die Unabhängigkeit von staatlichen, kommunalen oder Unternehmensstrukturen. Während traditionelle Stiftungen häufig über kein internes Kontrollorgan verfügen, ist dies bei den Bürgerstiftungen die Regel. Das Gesamtvermögen der deutschen Bürgerstiftungen beläuft sich derzeit auf über 60 Millionen Euro.

Bürgerstiftungen erfreuen sich in Fachkreisen und Medien großer Aufmerksamkeit. Bei potenziellen Stiftern, Spendern und ehrenamtlich Engagierten sind sie in den zehn Jahren ihres Bestehens zu einer attraktiven Alternative zu Vereinen und traditionellen Stiftungen geworden. Während die fachliche, überwiegend ehrenamtlich geleistete Arbeit der Bürgerstiftungen mit ihren Leseprojekten für Migrantenkinder oder der Förderung von Musikschulen oft einen etwas beschaulichen Eindruck erweckt, haben die Bürgerstiftungen einen innovativen Kern: sich selbst, denn sie sind eine institutionelle Innovation in der Organisationslandschaft der deutschen Bürgergesellschaft.

Die Bürgerstiftungen in Deutschland sind auf dem besten Weg, zu einer dauerhaften Erfolgsgeschichte zu werden. Zehn Jahre nach der Gründung der ersten Bürgerstiftung im nordrhein-westfälischen Gütersloh sind in mehr als 145 Städten, Gemeinden oder Regionen Bürgerstiftungen aktiv. Nach den USA und Kanada gibt es inzwischen in Deutschland die meisten Bürgerstiftungen weltweit. In keinem anderen Land entwickeln sie sich so dynamisch wie hierzulande.

Wie Hellmann (2006) in ihrer Untersuchung festgestellt hat, verdoppelte sich die Zahl der Bürgerstiftungen in Deutschland seit 1998 alle zwei Jahre. Im Jahr 2005 erreichte die Anzahl der neu ge- gründeten Bürgerstiftungen in Deutschland ihren bislang höchsten Wert. Bundesweit wurden im Jahr 2005 genau 37 Bürgerstiftungen gegründet. In allen Bundesländern mit Ausnahme des Saarlandes existieren Bürgerstiftungen. Die meisten gab es Ende August 2006 in Nordrhein-Westfalen (49 Bürgerstiftungen), es folgten die Bundesländer BadenWürttemberg (25) und Niedersachsen (24). Schlusslichter waren Brandenburg und Sachsen-Anhalt mit je einer Bürgerstiftung. Gut 27 Millionen Einwohner haben in Deutschland potenziellen $\mathrm{Zu}$ gang zu einer Bürgerstiftung, d. h. sie leben in einer Region, in der eine Bürgerstiftung aktiv ist. Das Gesamtvermögen der deutschen Bürgerstiftungen beläuft sich aktuell auf über 60 Millionen Euro.

Bürgerstiftungen sind jedoch vor allem deshalb interessant, weil sie eine institutionelle Innovation in der Organisationslandschaft der Bürgergesellschaft in Deutschland darstellen. Zwar gilt aufgrund des Fehlens einer gesetzlichen Definition jede Stiftung als Trägerin einer verselbstständigten Vermögensmasse und unterscheidet sich von einem Verein dadurch, dass sie in ihren Grundzügen nicht einem ständigen demokratischen Willensbildungsprozess ihrer Mitglieder unterworfen ist, sondern den bei Gründung wiedergegebenen Stifterwillen nachhaltig zu erfüllen hat.

Doch hat erstmals die Bürgerstiftung systematisch und konzeptionell die Stiftungsidee um assoziative Elemente einer Gemeinschaft von Stiftern ergänzt. Ihre Umsetzung findet dies bei der Bürgerstiftung in der Idee einer Stiftung von Bürgern für Bürger, was sich institutionell in Form der Stifterversammlung oder des Stifterrates und des Kuratoriums ausdrücken kann. Idealtypisch entscheiden hier Stifter und andere Organmitglieder über die grundsätzlichen Fragen der Stiftungsarbeit und nehmen wesentliche Kontrollaufgaben gegenüber dem für die operative Arbeit zuständigen Vorstand war. 
Für Entscheidungs- und Kontrollstrukturen von Unternehmen hat sich der Begriff der Corporate Governance eingebürgert. Corporate Governance kann als ein System verstanden werden, mit dem Organisationen geführt und überwacht werden, wobei manche Ansätze vor allem den optimalen Ausgleich zwischen verschiedenen Anspruchsgruppen einer Organisation betonen. Als Non-Profit-Governance hat das Thema inzwischen auch bei gemeinnützigen Organisationen Konjunktur (siehe Schuhen 2005).

\section{Governance-Modelle von Bürgerstiftungen}

Für die Gestaltung der GovernanceStrukturen von Bürgerstiftungen bieten die zehn Merkmale für Bürgerstiftungen des Bundesverbandes Deutscher Stiftungen eine Orientierung. Hierin heißt es in Punkt 3: "Eine Dominanz einzelner Stifter, Parteien, Unternehmen wird abgelehnt. Politische Gremien und Verwaltungsspitzen dürfen keinen bestimmenden Einfluss auf Entscheidungen nehmen. Punkt 10 führt aus: »Die interne Arbeit einer Bürgerstiftung ist durch Partizipation und Transparenz geprägt. Eine Bürgerstiftung hat mehrere Gremien (Vorstand und Kontrollorgan), in denen Bürger für Bürger ausführende und kontrollierende Funktionen innehaben. « (Bundesverband Deutscher Stiftungen 2006: 14 f.).

Die Ausgestaltung der GovernanceStrukturen in den Bürgerstiftungen hat sich in der Praxis vielfältig ausdifferenziert. Es zeichnen sich in den Bürgerstiftungssatzungen jedoch verschiedene Governance-Modelle ab, die bei einer Anzahl von Bürgerstiftungen Anwendung finden. Mit den hier vorgestellten vier Modellen soll keine Aussage über die Reichweite oder Verbreitung eines Modells getroffen werden. Vielmehr sollen Grundtypen von Entscheidungs- und Kontrollstrukturen in Bürgerstiftungen vorgestellt und anschließend diskutiert werden.

- Die »StadtStiftung Gütersloh - Die Bürgerstiftung « wurde als erste deutsche Bürgerstiftung 1996 gegründet. Sie ist auf Initiative von Reinhard Mohn, dem damaligen Vorstandsvorsitzenden der Bertelsmann Stiftung, entstanden und auf direkte Vorbilder und Erfahrungen aus den USA zurückzuführen. Die StadtStiftung Gütersloh gilt als klassi-

\section{Governance-Modell "Gütersloh"}

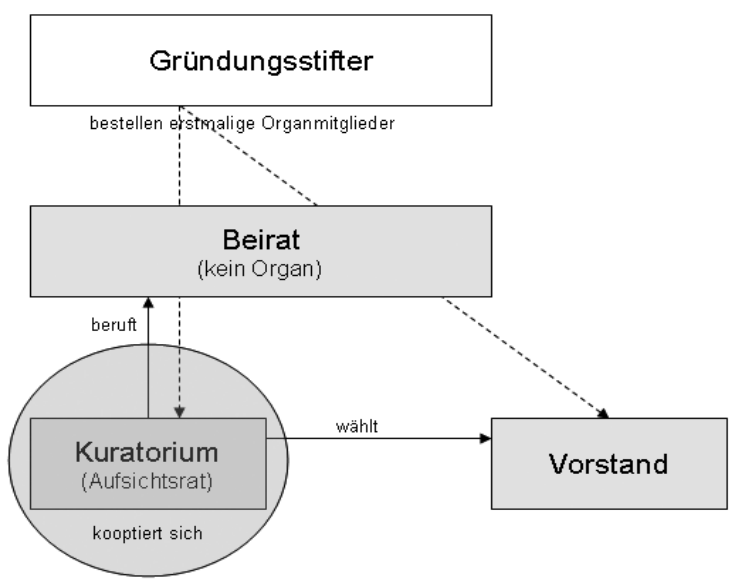

\section{Governance-Modell "Hannover"}

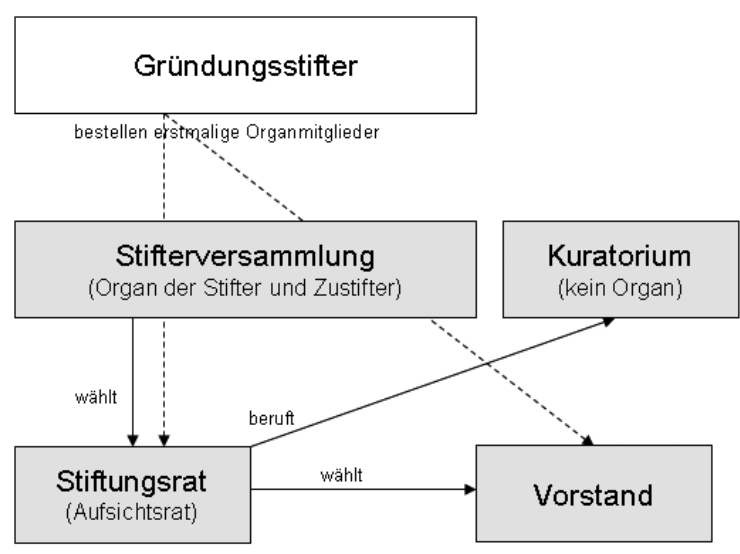

sche Top-down-Gründung. Sie verfügt laut Satzung über zwei Organe (vgl. Abbildung Governance-Modell »Gütersloh «). Die ersten Organmitglieder wurden im Stiftungsgeschäft durch den oder die Gründungsstifter benannt. Etwaige »Wahlen « im Vorfeld durch anwesende Stifter oder an der Stiftungsgründung Mitwirkende sind grundsätzlich möglich, aber nicht vorgeschrieben. Ein zwei- bis fünfköpfiger Vorstand führt die Geschäfte der StadtStiftung. Er wird seit der Satzungsänderung von 2006 vom Aufsichtsorgan, dem Kuratorium, gewählt (zuvor Kooptation). Das aus fünf bis zwölf Personen bestehende Kuratorium kooptiert seine Mitglieder. Es beruft auch die zehn bis 30 Mitglieder des Beirates, der die Funktion eines Ver- treters der Gütersloher Bürgerschaft hat und Kuratorium und Vorstand hinsichtlich der Verwirklichung des Stiftungszweckes berät und unterstützt. Neben diesen Gremien können nach der Satzung Arbeitsgruppen und eine Geschäftsführung eingesetzt werden. Das Kooptationsmodell der Erstfassung der Satzung der StadtStiftung Gütersloh fand und findet sich auch bei weiteren Bürgerstiftungen wie beispielsweise der Bürgerstiftung Parchim.

- Die Bürgerstiftung Hannover wurde ein Jahr später auf Initiative von Prof. Dr. Christian Pfeiffer, Direktor des Kriminologischen Forschungsinstitutes Niedersachsen, gegründet und orientiert sich ebenfalls direkt an US-amerikanischen Vorbildern. Die Gründung der 


\section{Governance-Modell "Aktive Bürgerschaft"}

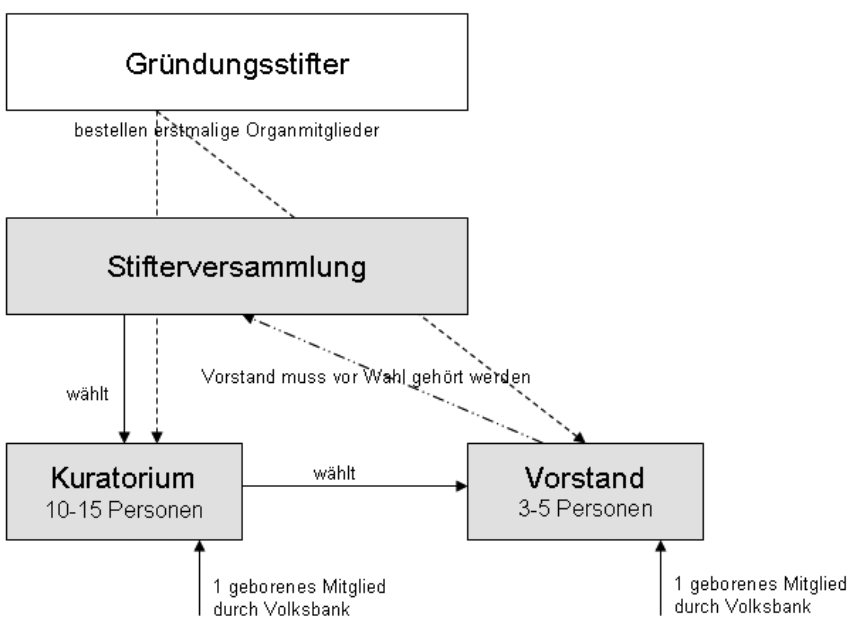

\section{Governance-Modell "Kommunal"}

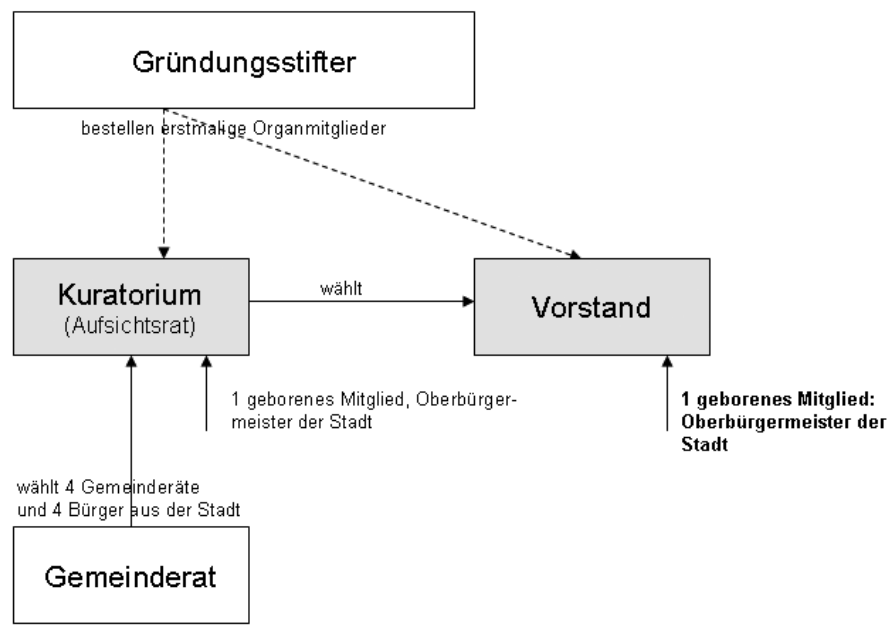

Bürgerstiftung Hannover wird in der Literatur als Bottom-up-Gründung beschrieben und zeichnet sich u. a. durch das Organ der "Stifterversammlung « aus (vgl. Abbildung Governance-Modell »Hannover «). Ihr gehören alle Stifter und Zustifter der Bürgerstiftung an, die eine finanzielle Zuwendung ab einer gewissen Höhe geleistet haben. Die Stifterversammlung wählt die Mitglieder des Stiftungsrates, der die Funktion eines Aufsichtsorgans hat. Die fünf bis zwölf Mitglieder des Stiftungsrates wiederum wählen die drei bis sieben Mitglieder des Vorstandes der Bürgerstiftung, der die Bürgerstiftung nach außen vertritt. Ebenfalls ist in der Satzung ein maximal 15-köpfiges Kuratorium vorgesehen, dessen Mitglieder vom Stiftungsrat berufen werden. Das Kuratorium hat für die Bürgerstiftung eine beratende Funktion. Fakultativ können Arbeitsausschüsse eingerichtet werden, ebenso eine durch den Vorstand zu bestellende Geschäftsführung. Die Satzung der Bürgerstiftung Hannover war Orientierung für viele Bürgerstiftungen. - Ebenfalls weite Verbreitung findet das Governance-Modell der Aktiven Bürgerschaft für Bürgerstiftungen, das der Struktur der Bürgerstiftung Hannover ähnelt. Auch hier wählen die Mitglieder der Stifterversammlung das zehn- bis 15-köpfige Kuratorium, dem die Funktion eines Aufsichtsorgans zukommt (vgl. Abbildung Governance-Modell
"Aktive Bürgerschaft «). Die Kuratoren wiederum wählen den Vorstand der Bürgerstiftung, der aus drei bis fünf Personen besteht. Die Besonderheit bei diesem Modell sind die geborenen Mitglieder in Aufsichts- und Exekutivorgan. Hiermit wird dem besonderen Engagement von Genossenschaftsbanken für Bürgerstiftungen Rechnung getragen. Dabei darf die Zahl der geborenen Mitglieder zugunsten einer Volksbank oder Raiffeisenbank nicht zu einer Dominanz des jeweiligen Organs führen. Derartige Governance-Strukturen haben beispielsweise die Bürgerstiftung Hellweg Region, die Bürgerstiftung Ostfalen und die Bürgerstiftung EmscherLippe Land. Das zweite Governance-Modell der Aktiven Bürgerschaft sieht wie bei der StadtStiftung Gütersloh keine Stifterversammlung vor. Die Mitglieder des Aufsichtsorgans kooptieren sich, wählen aber die Mitglieder des Vorstandes. Für die geborenen Mitglieder gelten die gleichen Bedingungen wie im ersten Modell. Solche Governance-Strukturen haben beispielsweise die Bürgerstiftung Region Mosbach, die Bürgerstiftung Mittelhessen und die Bürgerstiftung Rosenheimer Land. Auch hier ist die Einrichtung weiterer Gremien möglich, ebenso die Bestellung einer ehrenamtlichen oder hauptamtlichen Geschäftsführung.

- Schließlich findet eine Governance-Variante Verbreitung, die nicht mit den zehn Merkmalen für Bürgerstiftungen des Bundesverbandes Deutscher Stiftungen kompatibel ist. Problematisch ist bei diesem Modell sowohl a) die doppelte Organmitgliedschaft als auch b) die Dominanz der Bürgerstiftungsgremien sowie c) deren Benennung durch Externe (vgl. Abbildung Governance-Modell »Kommunal «). Am Beispiel der Bürgerstiftung Ludwigsburg wird nachfolgend eine typische kommunal dominierte Governance-Struktur dargestellt. So wird das neunköpfige Kuratorium als Aufsichtsgremium der Bürgerstiftung nicht durch ein Organ der Bürgerstiftung selbst gewählt oder berufen, sondern durch einen externen Dritten bestimmt, in diesem Fall den Gemeinderat der Stadt Ludwigsburg. Die hier vertretenen Gemeinderatsmitglieder wählen vier Kuratoren der Bürgerstiftung aus der Mitte des Gemeinderates sowie weitere vier Kuratoren, die nicht dem Gemeinderat angehören. Der Oberbürgermeister der Stadt gehört dem 


\section{Bürgerstiftungen und Soziales von Bernadette Hellmann und Elena Philipp}

Bürgerstiftungen bieten »alles aus einer Hand « für das Engagement in einer Stadt oder Region. Sie vergeben Fördermittel an gemeinnützige Organisationen und führen selbst Projekte in den Bereichen Jugend, Bildung, Kultur, Soziales, Gesundheit, Umwelt und Sport durch. Die aktuellen Schwerpunkte ihrer Aktivitäten liegen in den Bereichen Jugend (39\%), Kultur $(16 \%)$ und Soziales $(12 \%)$.

Vielerorts arbeiten Bürgerstiftungen mit sozialen Organisationen zusammen. Diese beteiligen sich als Stifter an einer Bürgerstiftung, erhalten Projektförderung oder initiieren gemeinsam mit der Bürgerstiftung eigene Projekte. Nachfolgend werden zwei Bürgerstiftungen vorgestellt, die gemeinsam mit sozialen Organisationen Aufgaben sozialer Arbeit übernehmen.

\section{Bürgerstiftung

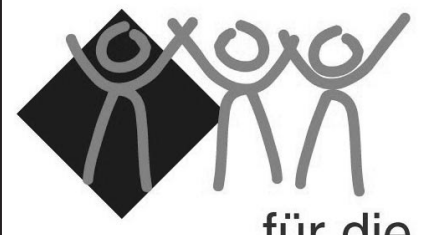 Region Mosbach} Bürgerstiftung für die Region Mosbach

Unter dem Leitspruch »Bürger helfen Bürgern« will die Ende 2002 gegründete Bürgerstiftung für die Region Mosbach bürgerschaftliches Engagement stärken, Eigeninitiative fördern und nachhaltige Projekte anstoßen. In 2005 konnten Projekte und Initiativen mit insgesamt 27.400 Euro unterstützt werden, darunter beispielsweise das Seminarprogramm »Erwachsen werden « mit den Lions-Clubs der Region Mosbach. Die offenen Hilfen der Johannes-Anstalten Mosbach, die Menschen mit Behinderung bei der Gestaltung eines eigenständigen und weitgehend selbstständigen Lebens begleitet, wurden bei der Umsetzung ihrer Ziele finanziell unterstützt.

Ein fortlaufendes Projekt der Bürgerstiftung für die Region Mosbach ist die Mosbacher Tafel, die Bürgerstiftung und Deutsches Rotes Kreuz 2004 gemeinsam ins Leben riefen. Der Tafelladen sammelt von Firmen ausgesonderte, noch zum Genuss geeignete Lebensmittel ein und gibt sie zu günstigen Preisen an Bedürftige ab. Die Bürgerstiftung vermittelte eine Ladeneinrichtung, Kasse, Waage etc. Auch stellte sie Kontakte zu Supermärkten, ortsansässigen Lebensmittelgeschäften und Metzgereien her und gewann diese als Zulieferer. Die Bürgerstiftung versteht sich als Wegbereiter und Unterstützer. Das Deutsche Rote Kreuz stellt die Räumlichkeiten bereit und setzt das Projekt mit zahlreichen ehrenamtlichen Helfern um.

Weitere Partner beteiligen sich an der Finanzierung. Gemeinsam mit DaimlerChrysler, dem DRK-Kreisverband und der Volksbank Mosbach finanzierte die Bürgerstiftung die Anschaffung eines Kühlfahrzeuges, das die Waren fachgerecht von den Märkten zur Tafel transportiert. Die Mosbacher Tafel hat inzwischen 450 Einkaufsberechtigungen an Bedürftige ausgegeben. Um die Arbeit der Tafel sicherzustellen, sind 30 Ehrenamtliche tätig, die die Lebensmittel abholen, sortieren und ausgeben.

Internet http://www.buergerstiftung-mosbach.de

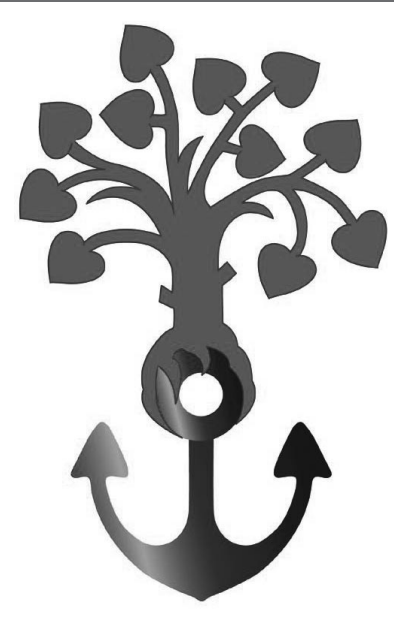

Bürgerstiftung

Tecklenburger Land

Die »Aktive Bürgerstiftung Tecklenburger Land für Mitmenschen in Not" wurde im Jahr 2000 als Sozialstiftung gegründet. Sie fördert das Ehrenamt und unterstützt Menschen in Notlagen. In der Satzung der Bürgerstiftung sind die Zwecke »Jugendhilfe, Altenhilfe und Wohlfahrtswesen « festgelegt.

Bislang half die Bürgerstiftung unter anderem bei der ehrenamtlichen Einrichtung einer Suppenküche und eines Beschäftigungsraumes für behinderte Menschen im Rentenalter, sie unterstützte die Ibbenbürener Tafel, entwickelte Integrationsmaßnahmen für ältere Aussiedler und engagiert sich in der Notfallseelsorge.

Die Bürgerstiftung Tecklenburger Land kooperiert dabei mit den Wohlfahrtseinrichtungen vor Ort. Der Sozialdienst Katholischer Frauen in Ibbenbüren, ein Fachverband im Deutschen Caritasverband, hat für die Bürgerstiftung die Geschäftsführung übernommen. Im Hauptförderprojekt der Bürgerstiftung, betitelt »Jung für Alt «, leisten Jugendliche ihr freiwilliges soziales Jahr derzeit in sieben Altenwohnhäusern im Tecklenburger Land, unter anderem bei AWO und Caritas. Im Jahre 2006 wurde die Bürgerstiftung Tecklenburger Land mit einem Preis für exzellente Projekte zum Thema Generationenverbundenheit ausgezeichnet. Die "Aktion Mensch" stellte daraufhin eine Anschubfinanzierung für drei Jahre bereit. Sie kommt dem am 1. November 2006 gestarteten Projekt »Alt für Jung « zugute, in dem Senioren jungen Menschen beim Übergang vom Schul- ins Berufsleben helfen.

Die Arbeit der Bürgerstiftung Tecklenburger Land steht auf einem soliden Fundament. 143 Stifter, zumeist Privatpersonen, hat die Stiftung schon gewonnen und konnte so seit ihrer Gründung bereits rund 150.000 Euro für Einzelfallhilfen und Projekte aufwenden. Durch die Organisationsform Bürgerstiftung ist sichergestellt, dass die Hilfe für Bedürftige stetig geleistet werden kann: Projekte dürfen nur aus den Erträgen des Stiftungskapitals oder mit Spenden finanziert werden. So können im Tecklenburger Land auch in Zukunft Menschen in Notlagen unterstützt werden.

Internet http://www.buergerstiftung-tecklenburgerland.de

Bernadette Hellmann ist Referentin für Bürgerstiftungen bei der Aktiven Bürgerschaft in Berlin.

E-Mail bernadette.hellmann@aktive-buergerschaft.de

Elena Philipp ist Projektassistentin bei der Aktiven Bürgerschaft in Berlin.

E-Mail elena.philipp@aktive-buergerschaft.de 
Kuratorium der Bürgerstiftung als geborenes Mitglied qua Amt an, ebenso dem zweiköpfigen Vorstand der Bürgerstiftung. Das zweite Vorstandsmitglied wird durch das Kuratorium gewählt.

Viele weitere solcher kommunalen »Bürgerstiftungen « verzichten gänzlich auf ein internes Aufsichtsorgan. Ähnliche Modelle finden sich beispielsweise bei der Bürgerstiftung Ulm und der Bürgerstiftung Fichtenberg.

\section{Diskussion}

Eine der Kernfunktionen von Bürgerstiftungen besteht in dem langfristigen Aufbau ihres Stiftungskapitals und der Förderung gemeinnütziger Aktivitäten vornehmlich durch die Erträge aus diesem Vermögen. Dadurch wird die Bürgerstiftung in die Lage versetzt, ihre Ziele

\section{Literatur}

Bundesverband Deutscher Stiftungen (2006): Bürgerstiftungen stellen sich vor. Berlin, Eigenverlag.

Hellmann, Bernadette (2006): Bürgerstiftungen in Deutschland. In: Aktive Bürgerschaft (Hg.): Länderspiegel Bürgerstiftungen. Fakten und Trends 2006. Berlin, Eigenverlag.

Hoelscher, Philipp/Eva Maria Hinterhuber (2005): Von Bürgern für Bürger? Bürgerstiftungen in Deutschlands Zivilgesellschaft. Berlin, Maecenata Verlag. Nährlich, Stefan (2005): Bürgerstiftungen als Ausdruck modernen Corporate Citizenships. In: Stefan Nährlich/Rupert Graf Strachwitz, Eva Maria Hinterhuber/Karin Müller (Hg.): Bürgerstiftungen in Deutschland. Bilanz und Perspektiven. Wiesbaden, VS-Verlag für Sozialwissenschaften, S. 53-64.

Rawert, Peter (2005): Bürgerstiftungen in Deutschland. Eine kritische Einführung aus juristischer Sicht. In Stefan Nährlich/Rupert Graf Strachwitz, Eva Maria Hinterhuber/Karin Müller (Hg.): Bürgerstiftungen in Deutschland. Bilanz und Perspektiven. Wiesbaden, VS-Verlag für Sozialwissenschaften, S. 39-51.

Schuhen, Axel (2005): Kontrollprobleme in Nonprofit-Organisationen und Lösungsansätze. In: Hopt, Klaus J./Hippel, Thomas von/Walz, Rainer W. (Hg.): Nonprofit-Organisationen in Recht, Wirtschaft und Gesellschaft. Tübingen, S. 221-241. unabhängig(er) von fremden Ressourcenzuflüssen zu verfolgen. Gleichzeitig stärken die Bürgerstiftungen damit langfristig die Fähigkeit der Bürgergesellschaft zur Selbstorganisation, was gegenwärtig insbesondere durch die hohe öffentliche Finanzierung gemeinnützigen Engagements nur eingeschränkt der Fall ist.

Vor diesem Hintergrund ist insbesondere die kommunale Dominanz von Bürgerstiftungen, wie sie im letzten GovernanceModell zum Ausdruck kommt, kritisch zu sehen. Dass die zu wählenden Personen in erster Linie nach parteipolitischer Mehrheit oder parteipolitischem Proporz ausgewählt werden, ist sicher keine unbegründete Annahme. Folgerichtig weisen auch die "Zehn Merkmale für Bürgerstiftungen « insbesondere auf die Wahrung der Unabhängigkeit von Politik und Verwaltung hin. Mit der institutionellen Verankerung der privatwirtschaftlichen und mitgliederbasierten Genossenschaftsbanken als Stifter in Bürgerstiftungen, verfolgt die Aktive Bürgerschaft das Ziel, die "private Seite " zu stärken (Nährlich 2005).

Während traditionelle Stiftungen häufig über kein internes Kontrollorgan verfügen, ist dies bei den Bürgerstiftungen die Regel. Diese interne formelle und fachliche Kontrolle begleitet die laufende Arbeit des Vorstandes und der Geschäftsführung einer Bürgerstiftung. Wesentliche Aufgaben liegen beispielsweise in der Bestellung von Prüfern für den vom Vorstand erstellten Jahresabschluss mit dem Bericht über die Erfüllung des Stiftungszwecks und dessen Genehmigung sowie natürlich in der Wahl und Abberufung der Vorstandsmitglieder.

Diese Kontrolle kann auch nicht durch die Aufsicht der Stiftungsbehörden und der Finanzämter ersetzt werden, da diese lediglich eine nachträgliche Rechtsaufsicht ausüben. Insofern kommt den Mitgliedern der Kontrollorgane eine hohe Verantwortung zu, die optimale Verfolgung der Stiftungsziele zu sichern. Inwieweit die Zusammensetzung der Kontrollorgane (Kooptation oder Wahl durch die Stifterversammlung) einen Einfluss auf den Erfolg der Arbeit von Bürgerstiftungen hat, ist bislang nicht untersucht worden. Aus Governance Perspektive problematisch sind solche Varianten, bei denen das Exekutivorgan die Mitglieder des Aufsichtsorganes wählt.

Dem besonderen Organ der Stifterversammlung, als partizipativem Element der
Einbindung einer breiten Anzahl von $\mathrm{Zu}$ stiftern, wie teilweise bei der Bürgerstiftung Nürnberg auch explizit auf "Zeitstifter « erweitert, sind durch das Stiftungsrecht Grenzen gesetzt (siehe dazu Rawert 2005). Die Bezirksregierung Karlsruhe hat als Stiftungsbehörde dazu ausgeführt, dass eine Stiftung keine Mitgliederorganisation sei und der Begriff "Stifterversammlung « aufgrund der Verwechslungsgefahr mit der Mitgliederversammlung nicht akzeptiert werden könne. Unter Berücksichtigung des Grundsatzes der sparsamen und wirtschaftlichen Verwaltung einer Stiftung sei jedoch, so die Bezirksregierung, die Errichtung eines »Stifterrates « nicht zwingend ausgeschlossen. Dabei soll dem Stifterrat aber grundsätzlich nur repräsentative und beratende Funktion zukommen, wobei es nach Auffassung der meisten Stiftungsbehörden akzeptabel ist, wenn aus der Mitte der Stifter die Mitglieder der Stiftungsorgane oder einzelner Stiftungsorgane gewählt werden.

In der Praxis ist bei den meisten Bürgerstiftungen mit einem Organ der Zustifter dieses auch überwiegend »nur « mit besonderen Informationsrechten ausgestattet. Die Partizipation der Stifter an »ihrer « Bürgerstiftung reicht dementsprechend von der Teilhabe an Informationen und dem Austausch mit anderen Stiftern bis zur Mitbestimmung, beispielsweise durch die Wahl von Organmitgliedern. Ob weitergehende Rechte einen zusätzlichen Anreiz zum Engagement bei einer Bürgerstiftung bieten würden, wird von Fachleuten unterschiedlich bewertet. Hoelscher und Hinterhuber (2005) kommen in ihrer Untersuchung zu einem eher negativen Ergebnis.

In der Praxis ist zumindest festzustellen, dass die Höhe der Zustiftung, die für eine Mitgliedschaft in der Stifterversammlung erforderlich ist, sich um Summen zwischen 500 und 1.000 Euro bewegt und damit eher den breiten Zugang ermöglicht als eine finanzielle Hürde darstellt. Selten sind dagegen Zustiftungen zwischen 10.000 und 20.000 Euro erforderlich.

Angesichts der generellen Entwicklungen im Non-Profit-Sektor, wie sie unter dem Stichwort »Non-Profit- Governance» diskutiert werden, können die Herausforderungen eher in der Verbesserung von Transparenz nach innen und außen sowie der Optimierung der internen Kontrolle der Qualität von Entscheidungen liegen als in der Einführung weitergehender Mitbestimmungsrechte für Zustifter. 